\title{
Absorption spectra and chromonic phase in aqueous solution of perylenetetracarboxylic bisimides derivatives
}

\author{
P. Camorani ${ }^{2}$, M. Furier, O. Kachkovskii ${ }^{1}$, Yu. Piryatinskiy, Yu. Slominskii ${ }^{1}$ and V. Nazarenko \\ Institute of Physics, NASU, 46 pr. Nauki, Kyiv 03039, Ukraine \\ ${ }^{I}$ Institute of Organic Chemistry, NASU, 5 Murmanskaya str., Kyiv 02094, Ukraine \\ ${ }^{2}$ University of Parma, Parco Area della Science, 7 A - 43100 Parma, Italy
}

\begin{abstract}
In this article we report the complex investigation of perylenetetracarboxylic bisimides derivatives in water solution. The results deal with all possible concentration in wide range of temperature. The experimentally observed of long wavelength maximums in absorption spectra with increasing of concentration can be attributed to decrease individual molecules of the dye and to the formation of aggregates. This fact is also responsible for the shift and form change of emission spectra.
\end{abstract}

Keywords: chromonic liquid crystal aggregate, fluorescence, absorption.

Paper received 26.06.01; revised manuscript received 04.07.01; accepted for publication 13.07.01.

\section{Introduction}

Lyotropic chromonic liquid crystals [1-3] (LCLC) are formed by flat polyaromatic molecules with ionic groups at the periphery that make them solvable in water. In water (or in a similar polar solvent such as glycerol), the molecules associate into aggregates, which then self-assemble into structures with long range orientational order, i.e. a liquid crystal. Because of the polyaromatic molecular structure, many chromonic materials are dyes. Aggregation involves interaction of the transition dipoles of the molecules and thus might strongly shift the position of the absorption band, as compared to the individual non-aggregated molecules. LCLCs have attracted considerable attention lately as a completely new type of liquid crystalline phases, different from the thermotropic and traditional lyotropic liquid crystals. The chromonic phases are lyotropic (solvent-based) materials, as opposed to the thermotropic (temperature-controlled) materials used in display industry. However, they are not traditional surfactant materials since they do not possess a flexible aliphatic (hydrophobic) chains.
Chromonic liquid crystal is a brilliant example when instilling a chemical compound into water we obtain the resulting materials with novel properties. Such self-assembly of dyes in aqueous solution has been proposed to manufacture polarizing and orienting films for thermotropic liquid crystal based display devices [4-6]. This application requires materials and processes to be well characterized and the same input must always produce the same output: working polarizers with a predictable specification. Before we can use the self-assembly techniques for making novel optical materials we need to make aggregation process controllable. In this article we report the complex investigation of perylenetetracarboxylic bisimides derivatives in water solution. The results deal with all possible concentration in wide range of temperature.

\section{Material and sample preparation}

Two types of dye were synthesized. There are $\mathrm{Na}$ (2327) and Ammonium (2334) salts of perylenetetracarboxylic bisimides. The dye 2327 has been synthesized in accordance with the following scheme: 

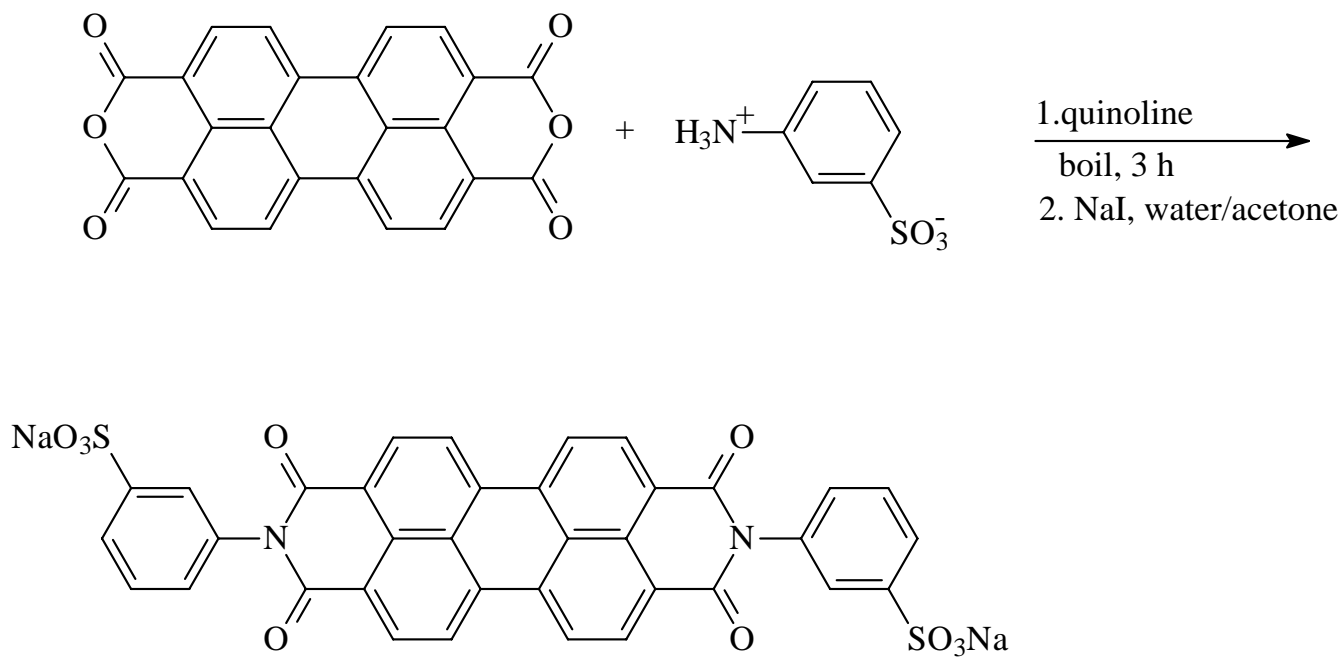

Similar approach was used to obtain ammonium salt: instead of $\mathrm{NaI}$ the $\mathrm{NH}_{3} \mathrm{I}$ was used. The both dyes have the same chromophore and thus possess similar absorption and fluorescence spectra. They possess different solubility in water.

The dyes were dissolved in distilled water by gently heating to about $40^{\circ} \mathrm{C}$. The solubility of this dye was good and concentration up to $10 \mathrm{wt}$. \% was achieved. For an each concentration used in the experiment the separate solution was made. The solutions were allowed to mature for about 24 hours in the dark. Oxygen was removed by bubbling nitrogen through them.

Stationary and time resolved fluorescence (FL) spectra were recorded using monochromator SPM-2 equipped with a photoelectric device. During the measurements, the accuracy was set at $0.2 \mathrm{~nm}$. The excitation was performed by nitrogen laser: wavelength of excitation was $337.1 \mathrm{~nm}$, duration of pulse was $10 \mathrm{~ns}$, frequency of pulses was $100 \mathrm{~Hz}$, power in pulse was $5 \mathrm{~kW}$.

The optimized equilibrium geometry of the molecule of the 2327 in the ground and excited states as well as the electron transition energies and oscillator strengths were obtained with the usage of the AM1 approximation (HyperChem package). Also, the transition energies and atomic charge redistribution upon excitation were calculated using the PPP approximation taking into consideration only $\pi$-electrons.

\section{Results}

The dyes exhibit poor solubility in methanol. The concentration of $10^{-4}$ wt. $\%$ was hardly achieved. The absorption spectrum (AB) of 2327 in methanol is shown in Fig. 1, curve a. The experimentally determined peak positions $523 \mathrm{~nm}, 487 \mathrm{~nm}, 457 \mathrm{~nm}$ are in accordance with CC-bonds vibration shift, that typically lies in the range $1300-1500 \mathrm{~cm}^{-1}$. FL spectrum demonstrates similar vibration structure relatively to $\mathrm{AB}$ (see Fig. 1 curve b) and shows mirror image symmetry between longest wavelength absorption band and the corresponding emission band. Remarkable is the small Stoke's shift between the FL and AB bends. Thus, the geometry of the $S_{0}$ and $S_{1}$ states is quite similar. The 2334 demonstrates similar absorption and fluorescence spectra in methanol solution. Low temperature absorption spectra have no difference for the 2327 as well as for the 2334. A dramatic difference in FL spectra occurs at low temperature. The maximum emission band becomes shifted to longer wavelength, $\lambda_{\max }=650 \mathrm{~nm}$, see curve $\mathrm{c}$ in Fig. 1. No visible changes were observed for the positive temperatures.

In Fig. 2 the absorption spectra of the 2327 in water solution are plotted. Up to the concentration of about $10^{-5} \mathrm{wt} . \%$ the spectra of dye are characterized by intense absorption band near $500 \mathrm{~nm}$ (curve a) and additional molecular peak at $539 \mathrm{~nm}$. Justification of molecular (M) and H-type dimer bands becomes apparent from the following experiment. The relative intensity of $\mathrm{M}$ - and $\mathrm{H}$ bands can be changed by varying the solvent composition $[7,8]$. Thus, addition of even small amount of alcohol diminishes H-band intensity, whereas M-band remains unchanged (see Fig. 3). With growing dye concentration the intensity of absorption is influenced by molecular aggregation. The aggregation process becomes more distinguishing for 2334 (see Fig. 4). The pronounced peaks, additional to molecular peak, at 584, 502, and $485 \mathrm{~nm}$ indicate that we can expect a number of aggregates with different lengths and/or of different types. We did not draw up the dependence of absorption spectra on temperature, since all these spectra have no distinguishing difference. The fluorescence spectra for aqueous solution of the both dyes become more complicate. These spectra are shown in Fig. 5 for the 2327. Further we will refer the 2334 dye only in case when measured parameters will become different from those of obtained from the 2327. The dilute water solution, concentration is about $10^{-5}$ wt. $\%$, demonstrates molecular emission for the both dye. The maximum of emission is located at 550 $\mathrm{nm}$ that is slightly red-shifted from the absorption of the 


\section{P. Camorani et al.: Absorption spectra and chromonic phase in aqueous ...}

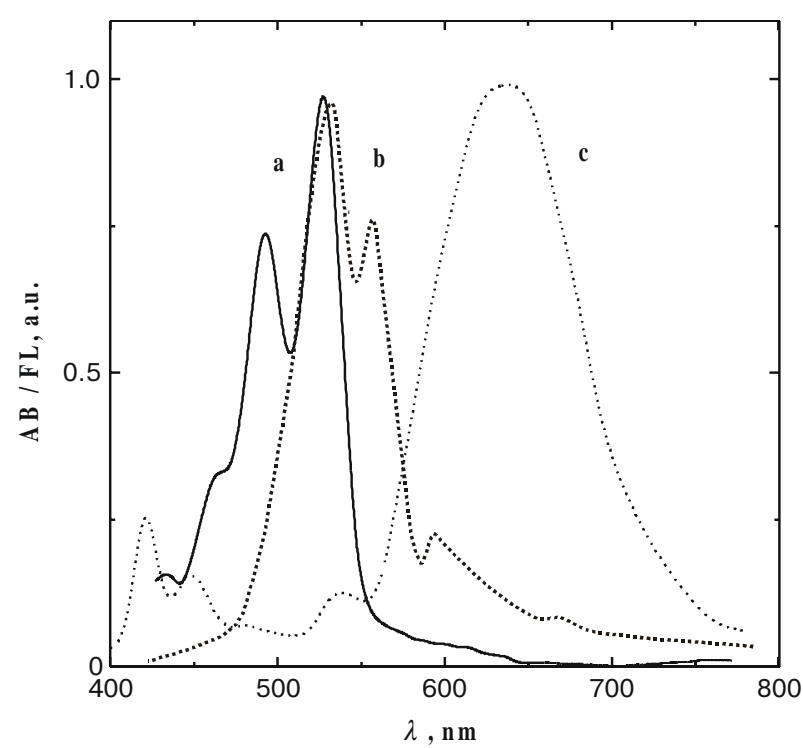

Fig. 1. Absorption and fluorescence spectra of the 2327 in methanol: a) absorption at $T=293 \mathrm{~K}$, b) fluorescence at $T=293 \mathrm{~K}$, c) fluorescence at $T=4.2 \mathrm{~K}$.

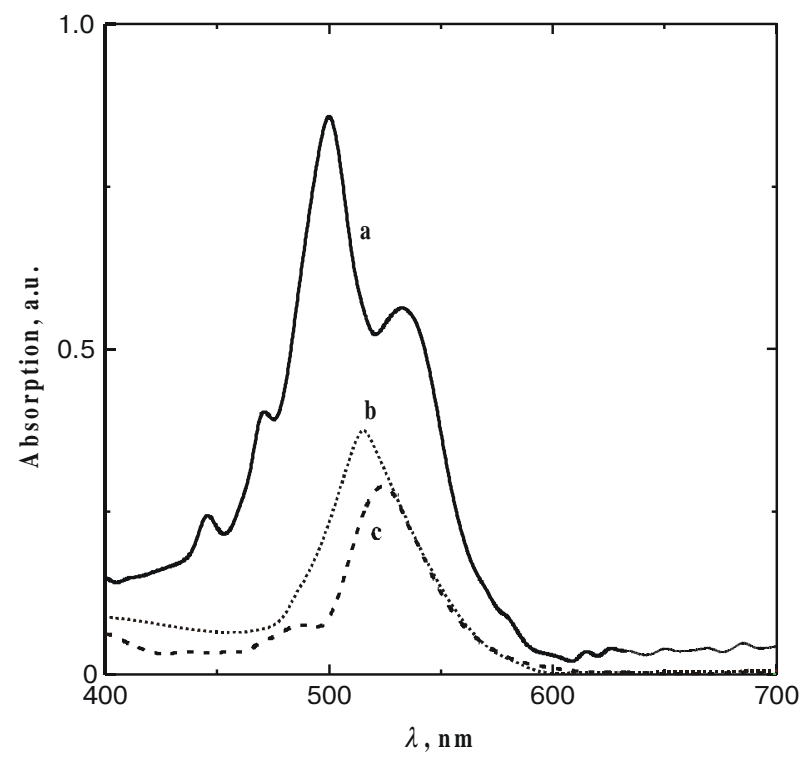

Fig. 3. Absorption spectra of the 2327 in water solutions: a) pure water, b) $3 \mathrm{wt} \%$ of alcohol is added, c) $10 \mathrm{wt} \%$ of alcohol is added. Concentration of dye is $10^{-5}$ wt. $\%$.

molecular band. The excitation spectrum for low dye concentration is shown in Fig. 6. Further increase in concentration leads to remarkable change in emission spectra. The molecular emission is diminished, whereas maximum of fluorescence appears around $700 \mathrm{~nm}$ as a wide structureless band. The transformation of the emission spectra occurs at the concentration of about $10^{-2} \mathrm{wt} . \%$. Starting from the concentration of about $10^{-2} \mathrm{wt} . \%$ one can observe additional low intensity peak around 600 $\mathrm{nm}$. The emission spectra with maximum at $700 \mathrm{~nm}$ were detected at the low temperature. The long wavelength emission band at the low temperature was observed for

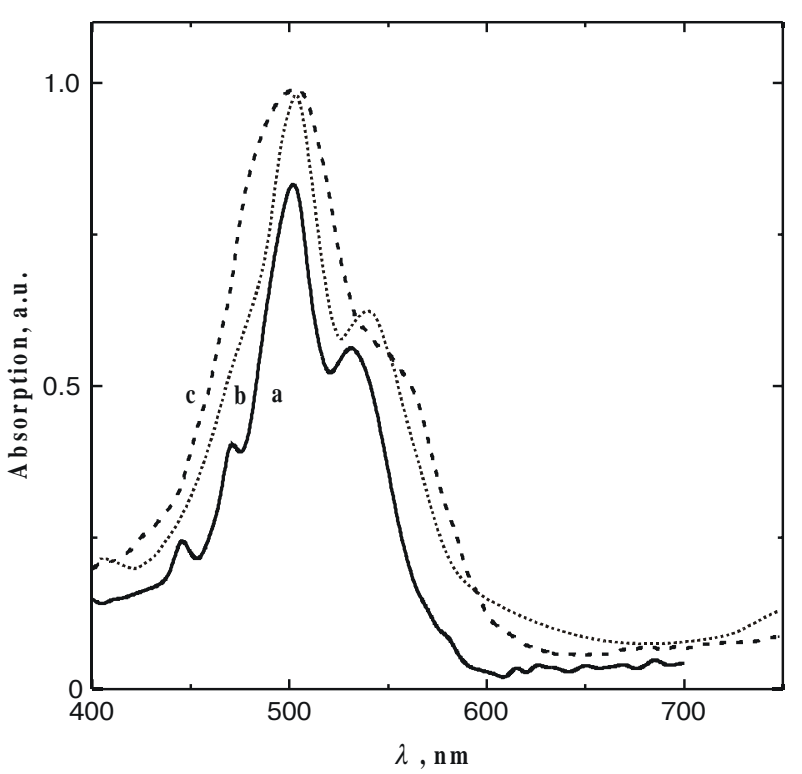

Fig. 2. Concentration dependence for the absorption of the 2327 in water at $T=293 \mathrm{~K}$ :

a) $10^{-5}$ wt. $\%$, b) $10^{-2}$ wt. $\%$, c) 10 wt. $\%$.

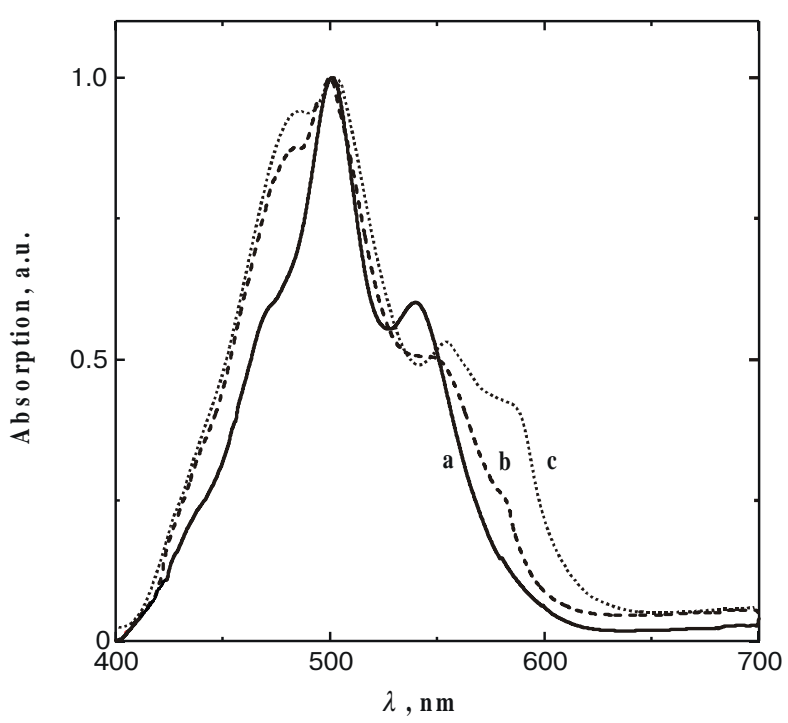

Fig. 4. Concentration dependence for the absorption of the 2334 in water at $T=293 \mathrm{~K}$ : a) $10^{-5}$ wt. $\%$, b) $10^{-2}$ wt. $\%$, c) 10 wt. $\%$.

all concentrations we measured. So, a transition we expect from concentration dependence might had the same origin as we observed from temperature dependence. The low temperature spectra are plotted in Fig. 7. The transition that occurs in the emission with temperature corresponds to the temperature of the transition water - ice.

\section{Discussion}

Now we will proceed to analysis of experimental data. We will begin from the nature of electron transition in the individual molecule of the 2327 [9-14]. The calcu- 
P. Camorani et al.: Absorption spectra and chromonic phase in aqueous ...

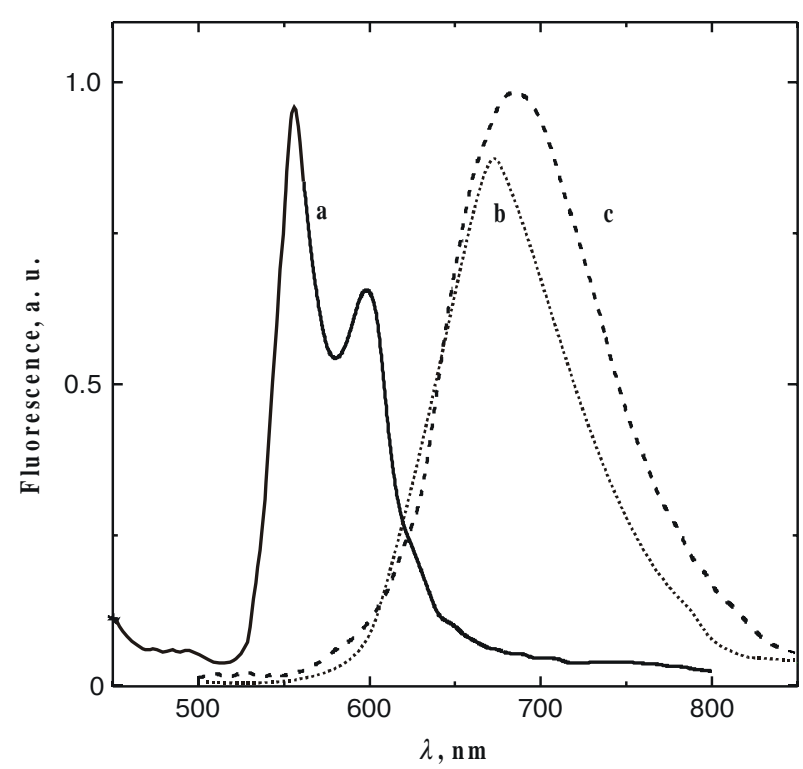

Fig. 5. Fluorescence spectra of the 2327 in water solution: a) $10^{-5}$ wt. $\%$, b) $10^{-1}$ wt. $\%$, c) film.

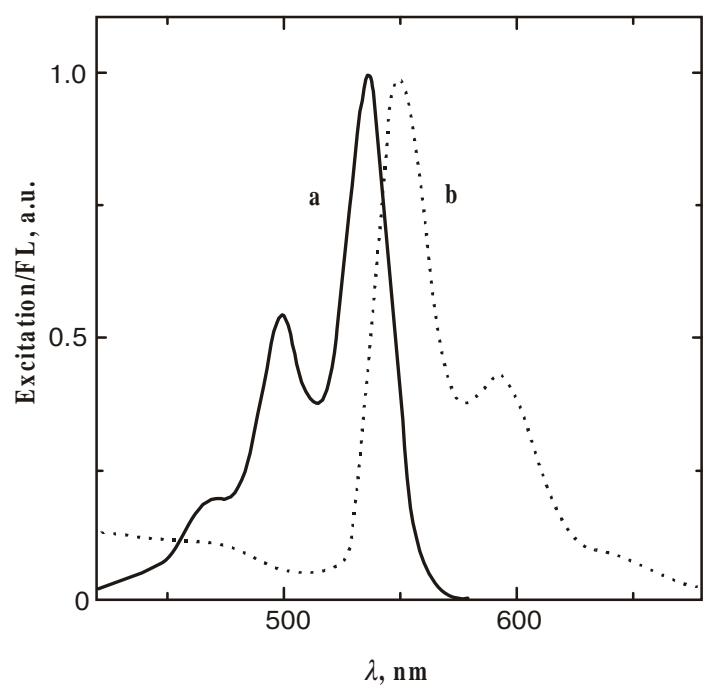

Fig. 6. Excitation (a)/fluorescence (b) spectra for $10^{-5}$ wt. $\%$ concentration of the 2327 in water.

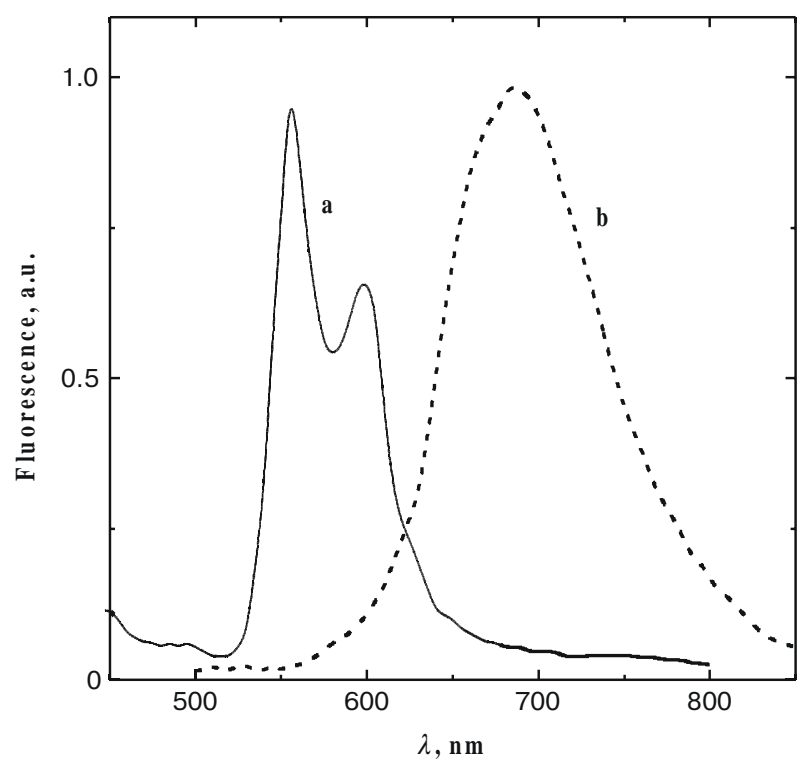

Fig. 7. Fluorescence spectra of the 2327 for $10^{-5}$ wt. \% concentration in water: a) $T=300 \mathrm{~K}$, b) $T=4.2 \mathrm{~K}$.

lated equilibrium geometry of the dye 2327 is presented in Fig. 8. It was found that the molecule is practically planar with the exception of two substituted phenyl residues which are out of the molecular plane on the equilibrium angle $\theta=46^{\circ}$. The calculated rotation barrier is shown in Fig. 9 for the both ground and excited states. The excitation leads to the practical all bond lengths increase slightly while the torsion angles remain practically the same. The molecular absorption and emission are observed for the 2327 in methanol solution, the emission spectra from low concentration water solution can also be attributed to molecular emission. The calculation shows that the molecular absorption band with maximal intensity as well as the maximum fluorescence band is induced by $\pi \rightarrow \pi^{*}$ electron transition. Since the highest occupied molecular orbital (HOMO) and the lowest unoccupied molecular orbital (LUMO) are almost completely localized on planar part of the molecules, the substituted residues do not practically influence on the en-

Ground state $S_{0}$

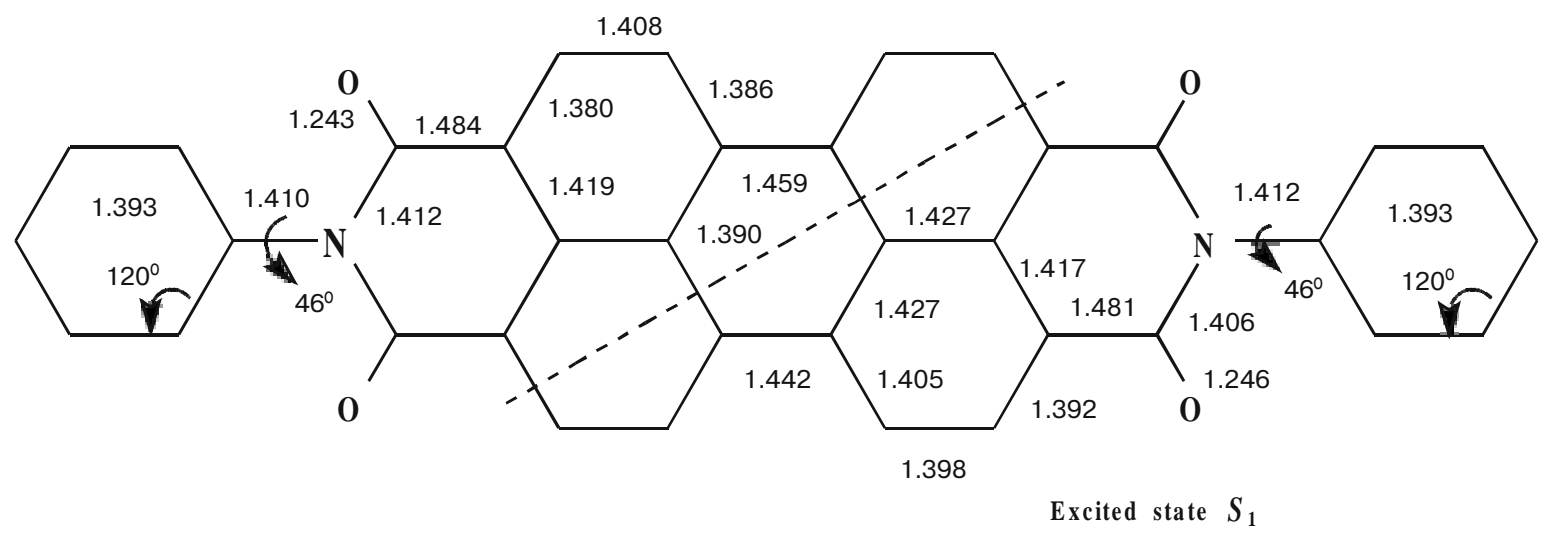

Fig. 8. Equilibrium geometry of the 2327 molecule in ground state (left part) and in excited state (right part). 


\section{P. Camorani et al.: Absorption spectra and chromonic phase in aqueous ...}

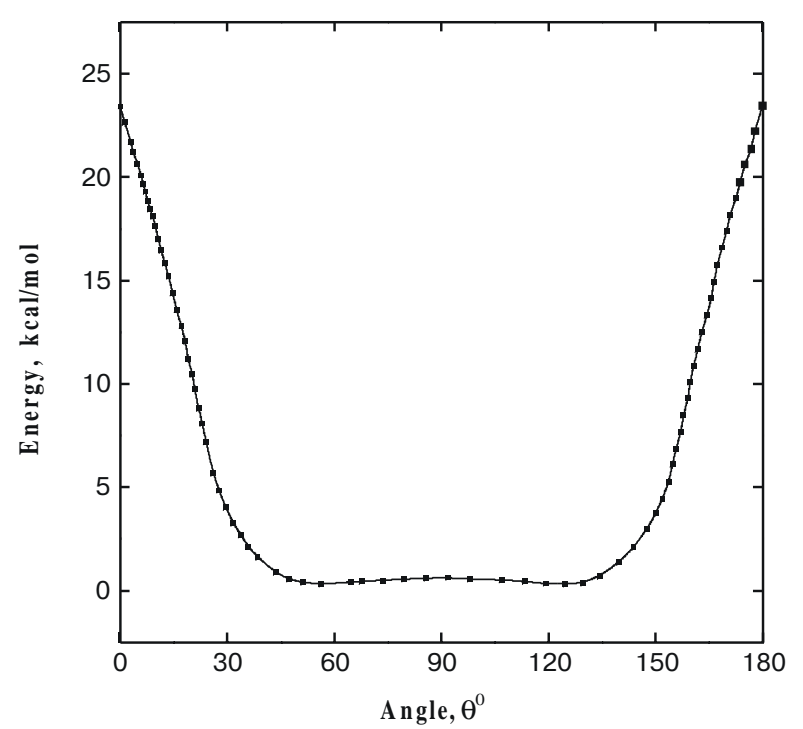

Fig. 9. Energy barrier for rotation of phenyl residues. Solid line was calculated for molecule of the 2327 that is in ground state, $S_{0}$, dashed line represents excited state, $S_{1}$.

ergy of lowest electron transition. This calculation agrees with our experimental data $\lambda_{\max }=525 \mathrm{~nm}$ and data obtained in $[9,10] \lambda_{\max }=523 \mathrm{~nm}$. The second transition in the 2327 is two times degenerated because the $\pi$-system has $D_{2 h}$ symmetry. Since the phenyl group rotated from the place of molecular, it leads to decrease of symmetry to $C_{2 v}$ or to $C_{2 i}$ and thus degeneration becomes partially removed. The calculation in both AM1 and PPP approximations demonstrates that these degenerated (quasidegenerated) transitions have a considerable higher energy than transition $S_{0} \rightarrow \mathrm{S}_{1}$. In this case the second and third peaks positions in absorption spectra might differ from the maximal absorption band on $80-120 \mathrm{~nm}$. So, the maxima at 525, 495 and $460 \mathrm{~nm}$, that are observed in absorption spectra of the 2327 in methanol solution, are the vibration transitions: $0 \rightarrow 1^{\prime}, 0 \rightarrow 1^{\prime} \ldots$

The distances between maximums, about $1400 \mathrm{~cm}^{-1}$, correspond to frequency of CC-bond oscillation in $\pi$-systems, $1300-1500 \mathrm{~cm}^{-1}$.

The transitions that correspond to the fluorescence spectra can be calculated in the range of quantum-chemical approximations at equilibrium geometry of molecule in an excited state. Since the lengths of chemical bonds does not significally change for excited state $S_{1}$, the Stoke's shift, calculated in AM1 approximation (56 configurations), $\Delta \lambda_{s}=16 \mathrm{~nm}$, is close to the experimental value, $\Delta \lambda_{s} \exp =11 \mathrm{~nm}$. The mirror symmetry of absorption and emission spectra of the 2327 in methanol, where the dye exists in molecular form, is also evidence of small geometrical change of the molecules in exciting state.

Proceeding from the methanol to the aqueous solutions of 2327, the absorption as well as emission spectra considerably change, even for low concentration solutions. Thus, for the concentration of about $10^{-5} \mathrm{wt} . \%$ the absorption spectrum is similar to that one obtained in methanol, but has different intensities of maximums as well as different distance between them. For example, the distance between first two maximums is $1150 \mathrm{~cm}^{-1}$ that is less than in methanol solution and less than frequency of CC-bond oscillation. With increase the concentration, the absorption band form does not noticeable change, except the distance between peaks demonstrates small growth. For high concentration as well as for film the spectra become wider and long wavelength peak transforms into shoulder. The additional and very important information on molecular and associate structures of dye can be obtained from fluorescence spectra. In our case the excitation of emission spectra (Fig. 6) registered for both emission peaks, 550 and $592 \mathrm{~nm}$, is almost identical to absorption spectra in methanol, i.e. reflects the molecular absorption.

The correct interpretation of experimental spectra is possible assuming that even for low concentration solution the both H-type of dimers and molecular form of the dye exist simultaneously. To analyze dimer and further aggregation process let us come back to molecular structure of the 2327. The phenyl residues which are out of the molecular plane on the equilibrium angle $\theta=46^{\circ}$, can almost freely rotate within a certain angle (see Fig. 9). The rotation of phenyl group plays important role in aggregation of molecules, since it limits the sliding of molecules and the only two types of dimer must be considered: a) the molecules are positioned exactly parallel with $\mathrm{SO}_{3}$ groups localized at the different side of the molecule (Fig. 10, parallel dimer); b) the molecules are rotated relative to each other (Fig. 10 rotated dimer). These dimers have a different ratio of intensities of peaks (see Table 1). According to the theory, all intensity of transition in the parallel dimer is concentrated in short wavelength region, while the long wavelength transition is strictly prohibited. In rotated dimers the both short wavelength and long wavelength transition are possible. Their intensities, $f_{1}$ and $f_{2}$, depend on the angle between vectors of dipole moments of the molecules. As it seen from Table 1 , increasing the angle up to $90^{0}$ gives $f_{1} / f_{2}=1$. Moreover, the value of splitting, $\Delta \lambda$ regularly decreases. For exact perpendicular position of molecules, the transition becomes degenerated and its energy equivalents to the energy of transition in monomer. So, the fact, that the excitation spectra for the $10^{-5} \mathrm{wt}$. $\%$ concentration solution is similar to the molecular absorption, demonstrates that solution contains the only parallel dimers (they do not fluorescent) which are in equilibrium with molecular form of the dye. It agrees with calculation showing that the parallel dimer is the most stable associate, since the molecules have maximal intersection. Additionally the parallel dimer is also stabilized by hydrogen bonds with four molecules of water, see Fig. 11. The energy of hydrogen bonds is $18 \mathrm{Kcal} / \mathrm{mol}$, that is considerable higher $\mathrm{kT}$.

The growth of concentration accompanies growth of a dimer number as well as creation of aggregates with a large number of molecules. The spatial obstacles (big $\mathrm{SO}_{3}^{-}$ groups) limit the creation of trimer, tetramer and so on with equivalent distance between molecules $3.4 \AA$. The only tetramer or higher associates with $2 \mathrm{~N}$ molecules and with alternative distances $3.4 \AA$ and $5.5 \AA$ are possible. In this case the translation unit is the parallel dimer (see Fig. 12). 
P. Camorani et al.: Absorption spectra and chromonic phase in aqueous ...

Table 1. Electron transition splitting (DI) in dimers (AM1, CI 2×2)

\begin{tabular}{cccccc}
\hline \hline \multicolumn{1}{c}{ Type } & Distance, $\mathrm{A}$ & Transition & $\Delta \lambda, \mathrm{nm}$ & $f$ & $f_{i} / f_{\text {monomer }}$ \\
\hline Monomer & - & $\mathrm{S}_{0} \rightarrow \mathrm{S}_{1}$ & - & 1.176 & - \\
\hline Parallel & 3.4 & $\mathrm{~S}_{0} \rightarrow \mathrm{S}_{1}$ & +16 & 0.000 & 0 \\
\hline & $\mathrm{S}_{0} \rightarrow \mathrm{S}_{2}$ & -35 & 2.273 & 2 \\
\hline Rotat. $30^{\circ}$ & & $\mathrm{S}_{0} \rightarrow \mathrm{S}_{1}$ & +29 & 0.140 & 0.12 \\
\hline & $\mathrm{S}_{0} \rightarrow \mathrm{S}_{2}$ & -25 & 2.344 & 1.99 \\
\hline $45^{\circ}$ & $\mathrm{S}_{0} \rightarrow \mathrm{S}_{1}$ & +26 & 0.308 & 0.26 \\
\hline & $\mathrm{S}_{0} \rightarrow \mathrm{S}_{2}$ & -18 & 1.955 & 1.66 \\
\hline $60^{\circ}$ & $\mathrm{S}_{0} \rightarrow \mathrm{S}_{1}$ & +18 & 0.546 & 0,46 \\
\hline & $\mathrm{S}_{0} \rightarrow \mathrm{S}_{2}$ & -9 & 1.614 & 1.37 \\
\hline $90^{\circ}$ & $\mathrm{S}_{0} \rightarrow \mathrm{S}_{1}$ & 0 & 1.171 & 1 \\
\hline & $\mathrm{S}_{0} \rightarrow \mathrm{S}_{2}$ & 0 & 1.171 & 1 \\
\hline \hline
\end{tabular}

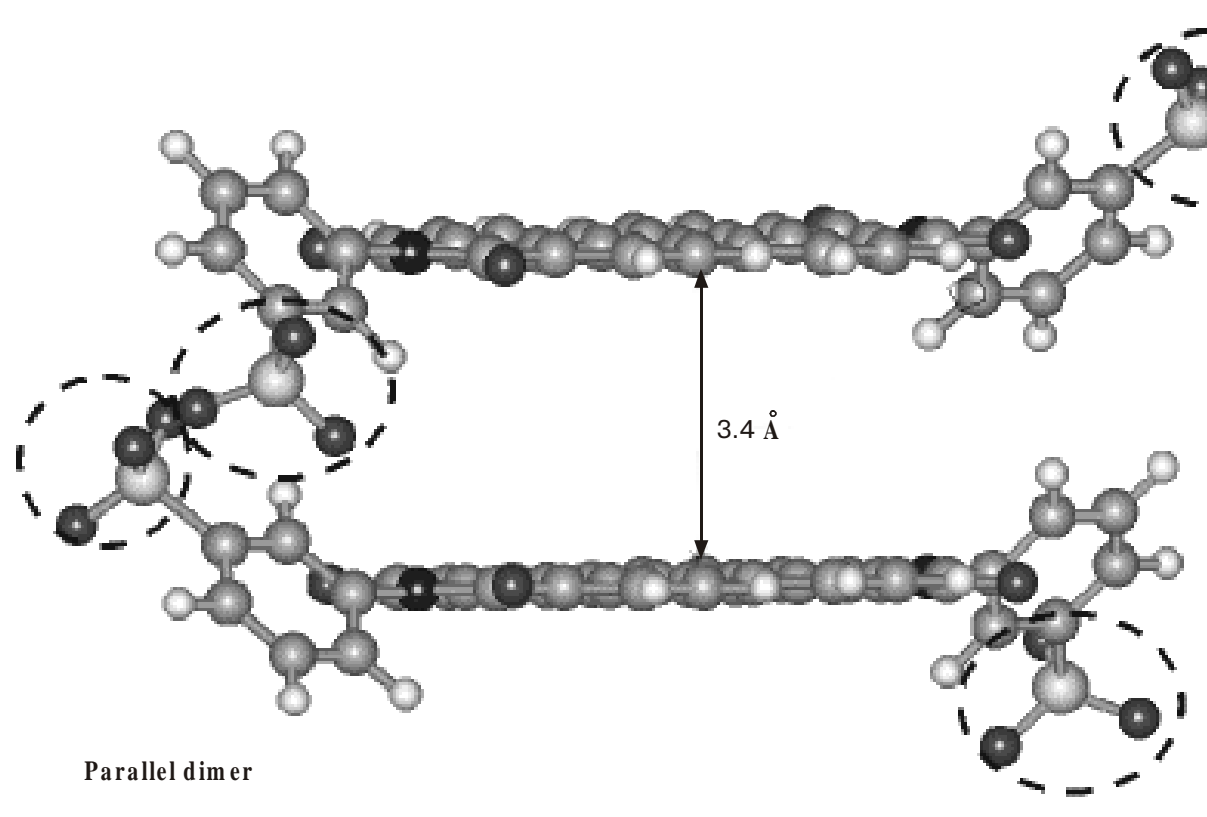

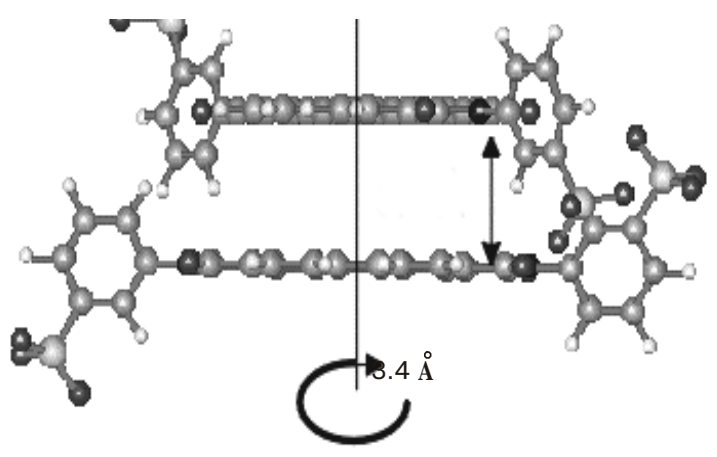

Rotated dimer

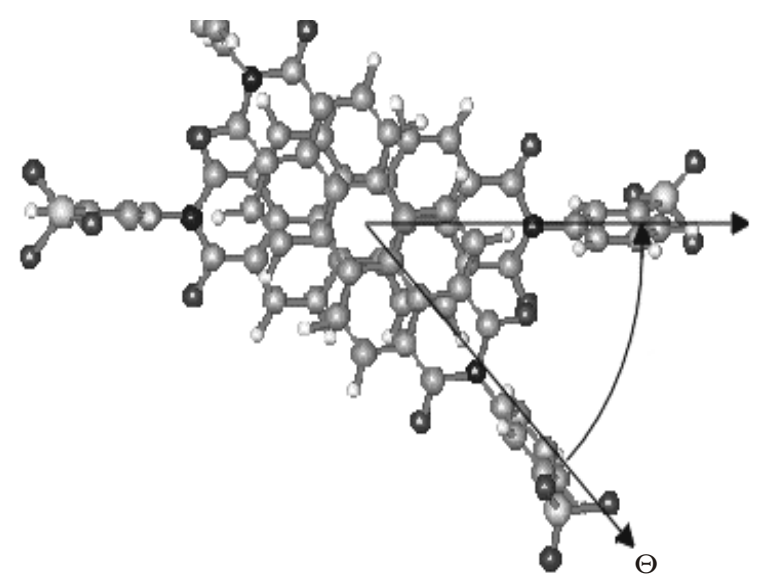

Fig. 10. Structure of dimers. 


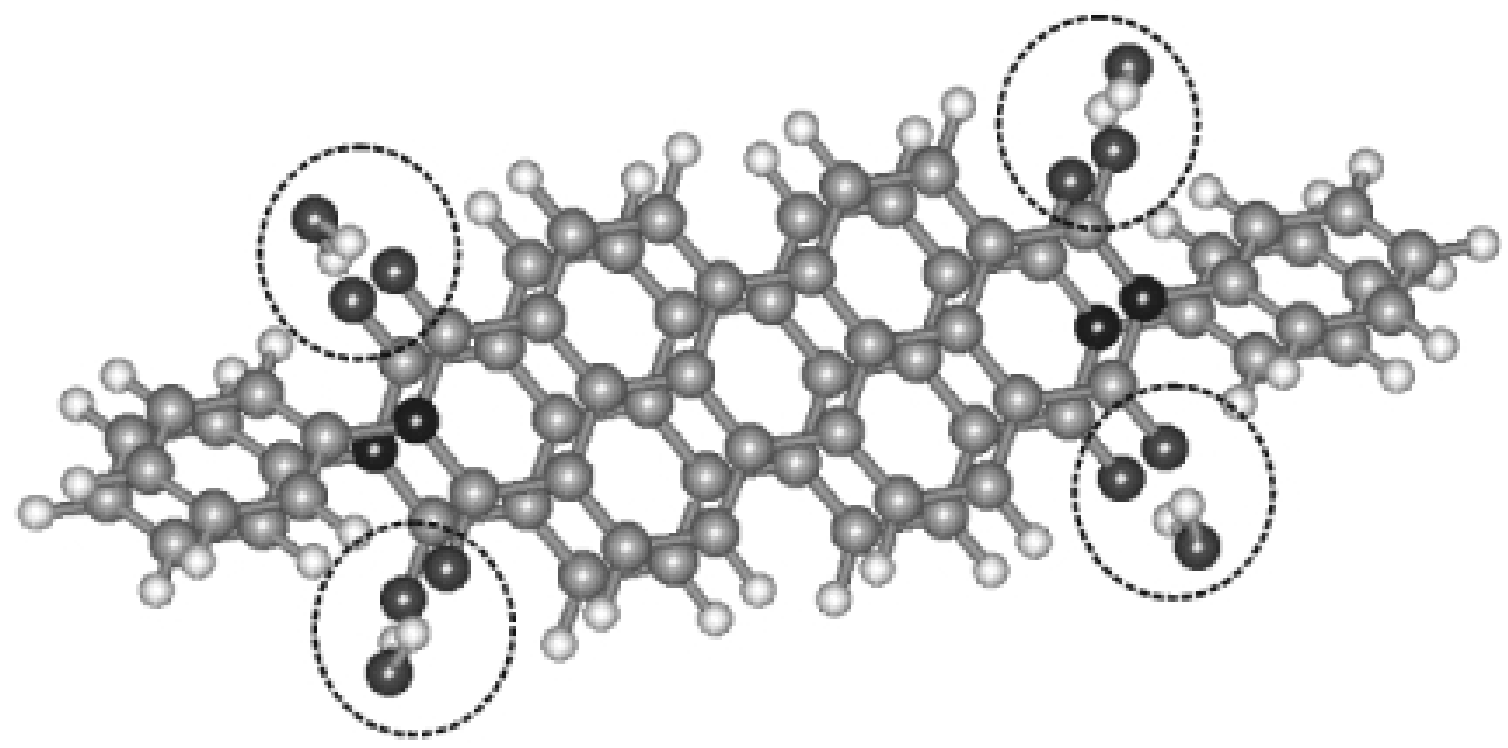

Fig. 11. Hydrogen bonds between dye and water molecules.

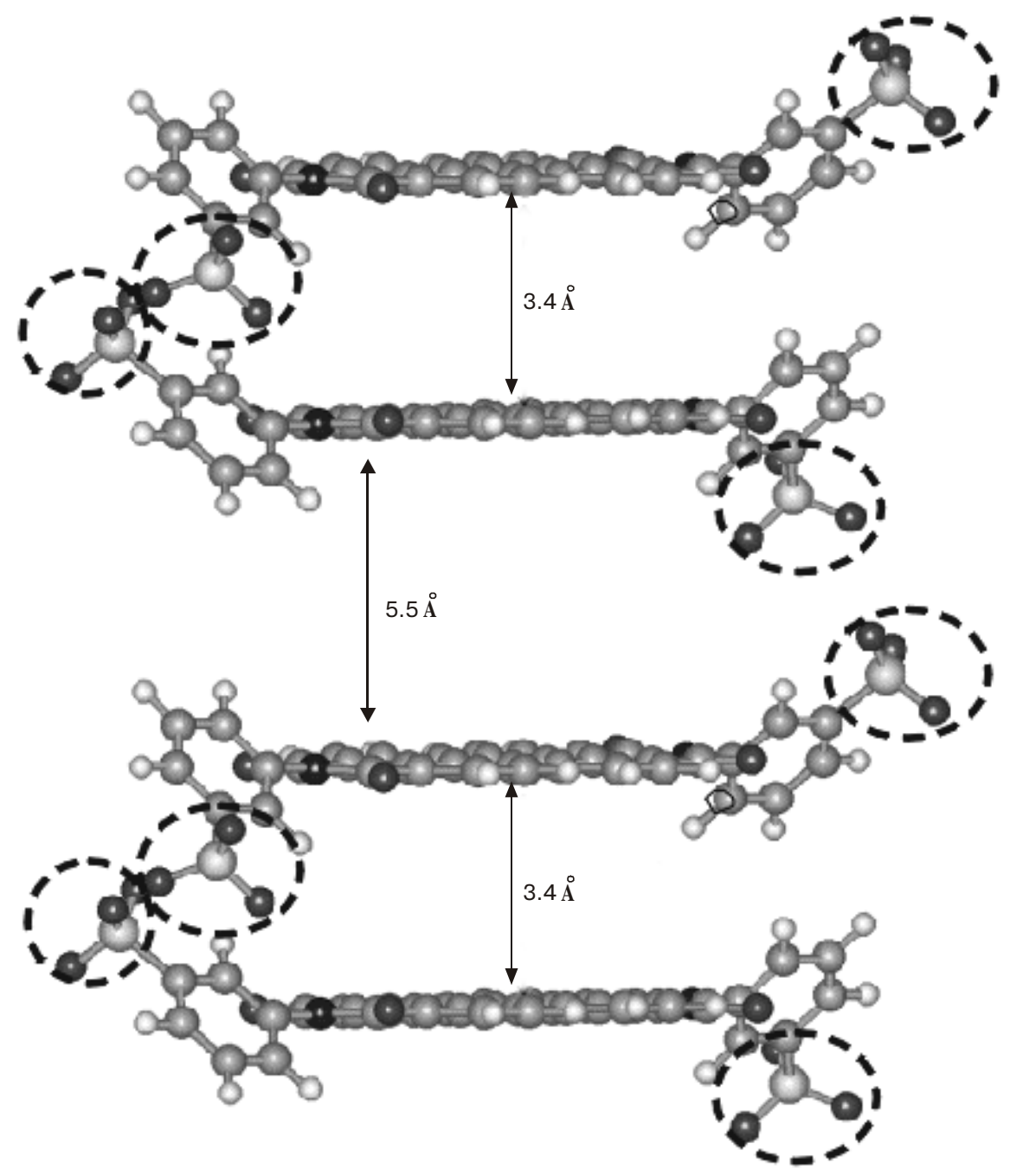

Fig. 12. Structure of parallel tertramer. 
P. Camorani et al.: Absorption spectra and chromonic phase in aqueous ...

Table 2. Electron Transition Splitting (DI) in Rotated Trimers (AM1, CI 3x3)

\begin{tabular}{|c|c|c|c|c|c|}
\hline Type & Angle, $\vartheta^{\circ}$ & Transition & $f$ & $\Delta \lambda, \mathrm{nm}$ & $f_{i} / f_{\text {monomer }}$ \\
\hline Monomer & - & $\mathrm{S}_{0} \rightarrow \mathrm{S}_{1}$ & 1.176 & - & - \\
\hline \multirow[t]{15}{*}{ Helix } & $15^{\circ}+15^{\circ}$ & $\mathrm{S}_{0} \rightarrow \mathrm{S}_{1}$ & 0.026 & +49 & 0.02 \\
\hline & & $\mathrm{S}_{0} \rightarrow \mathrm{S}_{2}$ & 0.120 & +12 & 0.10 \\
\hline & & $\mathrm{S}_{0} \rightarrow \mathrm{S}_{3}$ & 2.204 & -39 & 1.87 \\
\hline & $30^{\circ}+30^{\circ}$ & $\mathrm{S}_{0} \rightarrow \mathrm{S}_{1}$ & 0.195 & +34 & 0.17 \\
\hline & & $\mathrm{S}_{0} \rightarrow \mathrm{S}_{2}$ & 0.301 & +11 & 0.26 \\
\hline & & $\mathrm{S}_{0} \rightarrow \mathrm{S}_{3}$ & 2.221 & -25 & 1.89 \\
\hline & $45^{\circ}+45^{\circ}$ & $\mathrm{S}_{0} \rightarrow \mathrm{S}_{1}$ & 0.117 & +29 & 0.10 \\
\hline & & $\mathrm{S}_{0} \rightarrow \mathrm{S}_{2}$ & 0.984 & +3 & 0.84 \\
\hline & & $\mathrm{S}_{0} \rightarrow \mathrm{S}_{3}$ & 1.760 & 13 & 1.50 \\
\hline & $60^{\circ}+60^{\circ}$ & $\mathrm{S}_{0} \rightarrow \mathrm{S}_{1}$ & 0.044 & +30 & 0.04 \\
\hline & & $\mathrm{S}_{0} \rightarrow \mathrm{S}_{2}$ & 1.537 & 0 & 1.31 \\
\hline & & $\mathrm{S}_{0} \rightarrow \mathrm{S}_{3}$ & 1.175 & -6 & 1.00 \\
\hline & $90^{\circ}+90^{\circ}$ & $\mathrm{S}_{0} \rightarrow \mathrm{S}_{1}$ & 0.001 & +13 & 0.00 \\
\hline & & $\mathrm{S}_{0} \rightarrow \mathrm{S}_{2}$ & 0.903 & +7 & 0.77 \\
\hline & & $\mathrm{S}_{0} \rightarrow \mathrm{S}_{3}$ & 2.076 & -4 & 1.76 \\
\hline \multirow[t]{9}{*}{ Altern. } & $30^{\circ}-30^{\circ}$ & $\mathrm{S}_{0} \rightarrow \mathrm{S}_{1}$ & 0.156 & +39 & 0.13 \\
\hline & & $\mathrm{S}_{0} \rightarrow \mathrm{S}_{2}$ & 0.000 & +11 & 0 \\
\hline & & $\mathrm{S}_{0} \rightarrow \mathrm{S}_{3}$ & 3.393 & -38 & 2.88 \\
\hline & $60^{\circ}-60^{\circ}$ & $\mathrm{S}_{0} \rightarrow \mathrm{S}_{1}$ & 0.585 & +24 & 0.50 \\
\hline & & $\mathrm{S}_{0} \rightarrow \mathrm{S}_{2}$ & 0.000 & +15 & 0 \\
\hline & & $\mathrm{S}_{0} \rightarrow \mathrm{S}_{3}$ & 2.989 & -10 & 2.54 \\
\hline & $90^{\circ}-90^{\circ}$ & $\mathrm{S}_{0} \rightarrow \mathrm{S}_{1}$ & 0.000 & +14 & 0 \\
\hline & & $\mathrm{S}_{0} \rightarrow \mathrm{S}_{2}$ & 0.945 & +6 & 0.80 \\
\hline & & $\mathrm{S}_{0} \rightarrow \mathrm{S}_{3}$ & 2.065 & -4 & 1.76 \\
\hline
\end{tabular}

The rotated aggregates can be formed from separate molecules (see Fig. 13). Theoretically there are two possible aggregates: 1) helix, when each molecule turns by some angle forming a helix; 2) alternative, when an angle of rotation alternates as $\theta$ and $\theta^{+}$from one molecule to another. The calculated wavelength shift and oscillator strength for such model trimer are shown in Table 2, for tetramer - in Table 3. One can see that intensity of long wavelength transition grows with aggregation, particularly for alternative aggregates with $\theta= \pm 60^{\circ}$.Actually, there is a possibility for creation of rotated aggregates made from parallel dimers, but their long wavelength transitions are also prohibited.

With growing of concentration the FL spectra also demonstrate some changes. For high concentration solution the molecular emission is diminished, whereas maximum of fluorescence appears at $700 \mathrm{~nm}$ as a nonstructural band that can be approximated by Gaussian profile. The general feature of experiment in which we observed this structureless band is the hardness of surrounding. Thus, we determined this peak at the low temperature even for methanol solution. Low concentration water solution demonstrates this emission for all temperatures below zero whereas the molecular emission immediately appears with melting of ice. As far as it concerns the high concentration water solution, the molecules exist there in the form of aggregates which itself are rigid. It means that vibration channel to release excitation is partially limited for a separate molecule. In this case intermolecular charge transfer becomes dominant. For our dye molecules the nitrogen atom has a non-shared electron pair and plays a role of donor. The rest of molecular skeleton is an acceptor. Furthermore, the shift between absorption and emission bands, $4267 \mathrm{~cm}^{-1}$ is too big to be originated from molecular aggregation. So, the absent of monomer emission and energy shift between monomer absorption and emission peak are related to intermolecular charge transfer [14]. 
P. Camorani et al.: Absorption spectra and chromonic phase in aqueous ...

Table 3. Electron transition splitting (DI) in tetramers (AM1, CI 4x4)

\begin{tabular}{|c|c|c|c|c|c|}
\hline Type & Distance, A & Transition & $f$ & $\Delta \lambda, \mathrm{nm}$ & $f_{i} l f_{\text {momomer }}$ \\
\hline Monomer & - & $\mathrm{S}_{0} \rightarrow \mathrm{S}_{1}$ & 1.176 & - & - \\
\hline Parallel & $3.4-6.8-3.4$ & $\mathrm{~S}_{0} \rightarrow \mathrm{S}_{1}$ & 0.000 & +49 & 0 \\
\hline \multirow[t]{3}{*}{ by dimers } & & $\mathrm{S}_{0} \rightarrow \mathrm{S}_{2}$ & 0.000 & +44 & 0 \\
\hline & & $\mathrm{S}_{0} \rightarrow \mathrm{S}_{3}$ & 0.000 & -20 & 0 \\
\hline & & $\mathrm{S}_{0} \rightarrow \mathrm{S}_{4}$ & 4.499 & -38 & 3.82 \\
\hline Te-rotated & $3.4-3.4-3.4$ & $\mathrm{~S}_{0} \rightarrow \mathrm{S}_{1}$ & 0.011 & +52 & $\cong 0$ \\
\hline by dimers & & $\mathrm{S}_{0} \rightarrow \mathrm{S}_{2}$ & 0.010 & +45 & $\cong 0$ \\
\hline \multirow[t]{2}{*}{$60^{\circ}$} & & $\mathrm{S}_{0} \rightarrow \mathrm{S}_{3}$ & 1.180 & -17 & 1 \\
\hline & & $\mathrm{S}_{0} \rightarrow \mathrm{S}_{4}$ & 2.340 & -33 & 1.99 \\
\hline Helix & $30^{\circ}+30^{\circ}+30^{\circ}$ & $\mathrm{S}_{0} \rightarrow \mathrm{S}_{1}$ & 0.029 & +44 & $\cong 0$ \\
\hline \multirow[t]{3}{*}{ by mon. } & & $\mathrm{S}_{0} \rightarrow \mathrm{S}_{2}$ & 0.007 & +23 & $\cong 0$ \\
\hline & & $\mathrm{S}_{0} \rightarrow \mathrm{S}_{3}$ & 1.314 & -12 & 1.12 \\
\hline & & $\mathrm{S}_{0} \rightarrow \mathrm{S}_{4}$ & 0.453 & -41 & 0.38 \\
\hline Helix & $60^{\circ}+60^{\circ}+60^{\circ}$ & $\mathrm{S}_{0} \rightarrow \mathrm{S}_{1}$ & 0.014 & +33 & $\cong 0$ \\
\hline \multirow[t]{3}{*}{ by mon. } & & $\mathrm{S}_{0} \rightarrow \mathrm{S}_{2}$ & 0.283 & +12 & 0.24 \\
\hline & & $\mathrm{S}_{0} \rightarrow \mathrm{S}_{3,4}$ & 1.391 & -10 & $1.18 \times 2$ \\
\hline & & $\mathrm{S}_{0} \rightarrow \mathrm{S}_{5,6}$ & 2.327 & -10 & $1.98 \times 2$ \\
\hline Altern. & $60^{\circ}-60^{\circ}+60^{\circ}$ & $\mathrm{S}_{0} \rightarrow \mathrm{S}_{1,2}$ & 0.029 & +22 & $\cong 0$ \\
\hline \multirow[t]{3}{*}{ by mon. } & & $\mathrm{S}_{0} \rightarrow \mathrm{S}_{3,4}$ & 0.777 & +21 & $0.66 \times 2$ \\
\hline & & $\mathrm{S}_{0} \rightarrow \mathrm{S}_{5}$ & 0.306 & +4 & 0.26 \\
\hline & & $\mathrm{S}_{0} \rightarrow \mathrm{S}_{6}$ & 2.333 & -19 & 1.98 \\
\hline
\end{tabular}

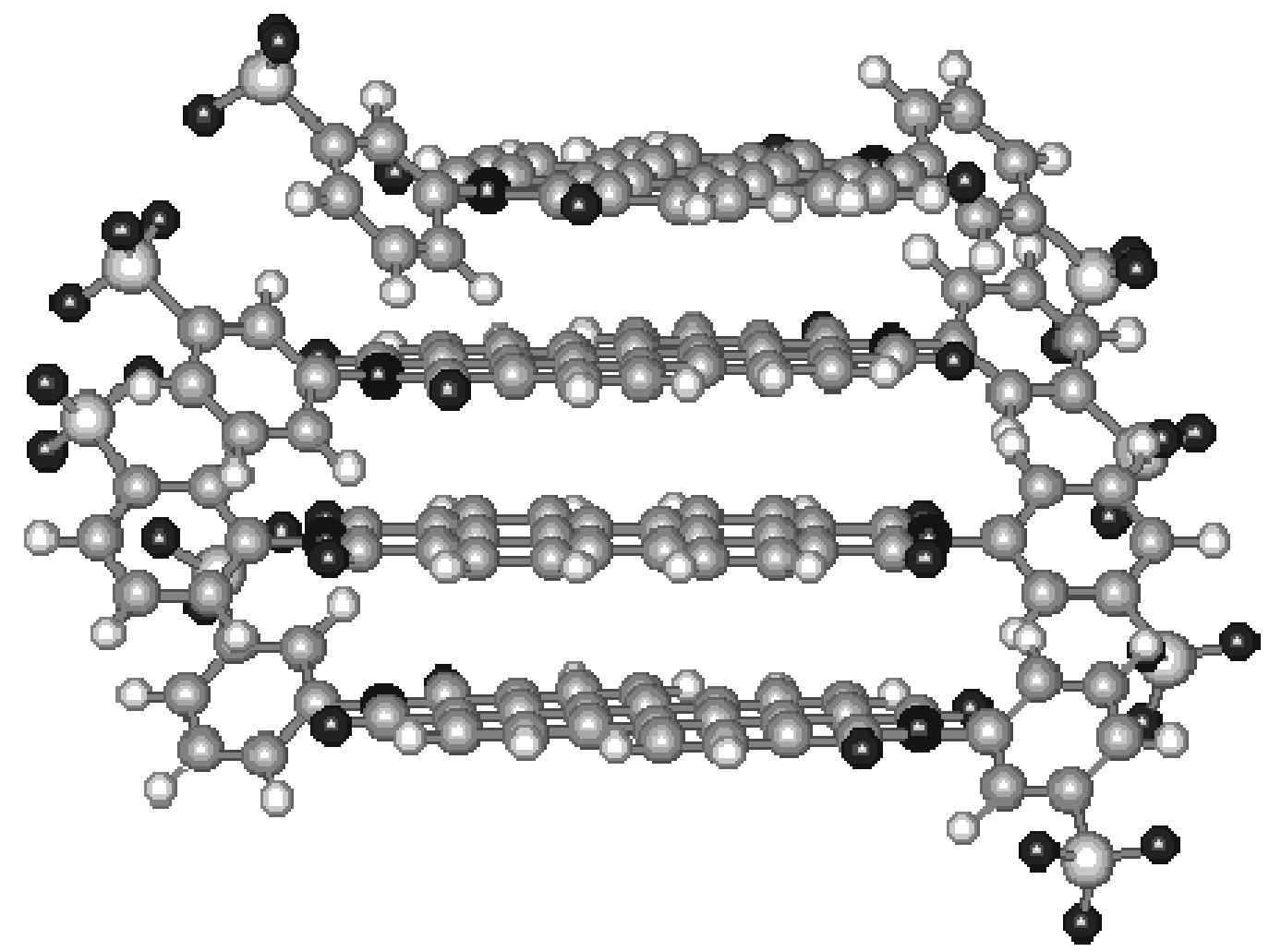

Fig. 13. Structure of rotated tetramer. 


\section{Conclusions}

The experimental spectra and quantitative comparison of energy shifts calculated for aggregates do not permit to make a clear choice between possible structures of aggregates. The maximum absorption band at $500 \mathrm{~nm}$ in low concentration water solution belongs to parallel or $\mathrm{H}$-type of dimer, the maximum at $525 \mathrm{~nm}$ belongs to the monomer. The possible existence of rotated dimer probably leads to uniform wideness of absorption spectra. The experimentally observed long wavelength maxima in the absorption spectra of the 2327 and the 2334 with increasing concentration can be attributed to decreasing number of individual molecules of the dye and to the formation of the rotated aggregates. This fact is also responsible for the shift and form change of emission spectra $(580-600 \mathrm{~nm}$ emission maximum).

\section{Acknowledgments}

We acknowledge helpful discussion with A. Nych, V. Pergamenshchik, O. Lavrentovich, M. Fontana and T. Schneider. We also acknowledge B. Blonskiy for supply of helpful references on formation and self-ordering of structures in semiconductors. The work was supported by STCU grant \#2025 and INTAS grant \#30234.

\section{References}

1. J. Lydon, Chromonics, in: Handbook of Liquid Crystals, (Wiley-VCH, Weinhein) vol. 2B, p. 981 (1998).

2. J. Lydon., MCLC 64, p. 19 (1980).

3. T. K. Attwood, J. E. Lydon, MCLC 108, p. 349 (1984).

4. T. Sergan, T. Schneider, J. Kelly, and O. D. Lavrentovich, Liq. Cryst. 27, p. 567 (2000).

5. T. Schneider and O. D. Lavrentovich, Langmuir, 16, p. 5227 (2000).

6. Yu. P. Piryatinski, V. G. Nazarenko, and O. V. Yatsun, Technical Physics Letters, 25(10), p. 761 (1999).

7. E. Daltrozzo, G. Scheibe, K. Gschwind and F. Haimer, Photographic Science and Engineering 18, p. 141 (1974).

8. Douglas L. Smith, Photographic Science and Engineering 18, p.309 (1974).

9. A. Rademacher, S. Markle and H. Langhals, Chem. Ber. 115, p. 2927 (1982).

10. H. Langhals and W. Jona, Angew. Chem. Int. Ed. 37, p. 952 (1998).

11. H. Langhals, H. Jaschke, U. Ring and P. von Unold, Angew. Chem. 111, p. 143 (1999).

12. H. Langhals and S. Kirner, Eur. J. Org. Chem. 2000, p. 365380 .

13. F. O. Holtrup, G. R. J. Muller, H. Quante, S. de Feyter, F. C. de Schyver and K. Mullen, Chem. Eur. J. 3, p. 219 (1997).

14. Karl-Heinz Koch and Klaus Mullen, Chem. Ber. 124, p. 2091 (1991) 